\title{
No regrets? Measuring the career benefits of a psychology placement year
}

\author{
Elisabeth Moores and Peter Reddy \\ Psychology, School of Life and Health Sciences, Aston University, Aston Triangle, \\ Birmingham, B4 7ET \\ e.j.moores@aston.ac.uk \\ tel: +44 (0)121 2044070 \\ fax: +44 (0)121204 4090
}

\begin{abstract}
We report an analysis of whether a psychology placement year provides a significant benefit to graduates' careers. Destination of Leavers from Higher Education (DLHE) survey data six months post-graduation suggested that placement programme graduates across the university are more likely to be (i) in work and (ii) in graduate level jobs. For psychology, the association between graduates' placement status and employment status at six months post graduation was not significant overall. However, when analyses were split by degree classification obtained, it was shown that amongst those graduates with 2.1 classification degrees reporting themselves as working, more placement programme vs. non-placement programme graduates had obtained graduate level jobs (63\% vs. 33\%). In 2.2 graduates there was no significant association. This pattern persisted in the data from a survey of psychology alumni (from 18 months to six and a half years post graduation). Psychology placement programme alumni were more satisfied with their careers even when ethnicity, gender, degree classification and entry year were taken into account. They also earn more, although not when background factors are taken into account. This
\end{abstract}


study was therefore able to show some measurable and persistent effects of a psychology placement year, although whether the benefits can be claimed to outweigh the costs is inconclusive. Limitations and implications are discussed.

\section{Introduction}

Graduate employability continues to be high on the UK government agenda. Work placements are generally accepted as a good way to increase employability, have long been a feature of vocational courses, and the benefits seem clear. From an analysis of 258 in-depth interviews with managers, and graduate and non-graduate employees, Harvey, Moon and Geall with Bower (1997) confirmed the value that employers place on work experience of graduates. Similarly, Blasko with Brennan, Little, and Shah (2002) found "measurable employment benefits" (p7) for all graduates who had a 'substantial' (9 months or more) period of work experience during higher education. This included having a job the graduate does not feel overqualified for, a higher salary (males only) and greater job satisfaction (males only). Interestingly, this was found to be especially true for graduates from non-vocational courses (although it did not seem to hold true for mature student graduates), perhaps due to a greater need for work experience. Cranmer (2006) suggested a limited impact of employability skills teaching within the classroom, but found a positive effect of structured work experience and employer involvement in degree course design and delivery.

Across Aston University around $70 \%$ of students take a voluntary or compulsory placement year between their second and final years; in certain vocational subjects e.g. Pharmacy and Optometry, the main work experience is gained post-graduation 
in a pre-registration year. In psychology, the optional placement year (minimum of 30 weeks work experience) is becoming increasingly popular; around $75 \%$ of potential placement year students took one in 2010. Students find their own placements with support and assistance. About half of those taking a sandwich year choose psychology-specific placements and work in an apprentice role with a professional psychologist, often in a research or clinical setting. The remainder do more broadly psychology related work, for example in human resource management. Many students aim for a career in clinical psychology, but to qualify as a clinical psychologist is a long process. To obtain a place on a clinical doctorate is exceptionally competitive and normally requires a period of work experience, usually under the supervision of a qualified NHS clinical psychologist as a psychology assistant. The competition for clinical training is such that even to obtain a post as a psychology assistant is highly competitive, despite the fact that the pay levels do not generally compare favourably with other graduate level jobs (£16-£18K per annum). In addition, it is probably realistic to suggest that even to get a psychology assistant post, relevant (usually voluntary) work experience is a significant advantage.

Reddy and Moores (2006) measured the benefits of a psychology placement year at Aston University and found benefits in terms of final year academic performance, staff ratings of transferable skills and career direction. Auburn, Ley and Arnold (1993) found that psychology graduates who had taken placements rated their first employment experience as more satisfactory than those who had not. However, this difference was not apparent when placement programme graduates were compared to students who graduated in the same year, suggesting that the results may have been at least partially related to economic climate rather than, or as well as, 
undergraduate experience. Moreover, in a report to HEFCE, Mason et al (2003) found that across five different University subjects (not including psychology), any benefits of sandwich placement participation that were apparent six months postgraduation, were no longer significant one to three years later. However, they also confirmed the importance of taking into account subject-related differences in any evaluation of employability skills. Measuring the benefit of a placement year in both the short and longer term is particularly crucial for subjects such as psychology where placement positions are often unpaid. A placement year delays graduation and employment for a year and can be expensive for the student and his or her parents. Thus, Mason et al's findings raises questions as to whether a psychology placement year is really worth undertaking. On the one hand a placement year may, through several mechanisms, benefit the student on graduation so that clinical work or a place on a graduate programme is achieved; thus having a determining influence on career direction and success. On the other hand Mason et al's findings may suggest so that the placement year benefit may be a 'sun tan effect' - fading over time so that placement and non-placement graduates become indistinguishable on a variety of measures.

Psychology graduates are employed in a wide variety of sectors and at different pay levels. They also have attempted career paths into the psychology professions that may include periods of junior or non-graduate (for example care assistant) roles. Because of this, it is difficult to define career 'success' in terms of employment position or pay alone. Career success encompasses satisfaction with a career and career progress. Turban and Dougherty (1994) investigated career attainment in terms of dollars earned, but also perceived career success - in general, in relation to 
peers, and accounting for age. Part of Greenhaus, Parasuraman and Wormley's (1990) evaluation of career outcomes looked at career satisfaction generally, as well as satisfaction in terms of meeting career goals, goals for advancement, goals for skill development and goals for income. Reddy and Moores (2006) looked at knowledge of career direction with recent psychology graduates and progress towards career goals.

The DLHE (Destinations of Leavers from Higher Education) survey is a UK wide survey run by the Higher Education Statistics Agency six months following graduation. Some of the results of this survey are coded using a system for classifying graduate occupations suggested by Elias and Purcell (2004). This system classifies jobs into five categories: (i) traditional graduate occupations (the established professions e.g. solicitors, medical practitioners); (ii) modern graduate occupations (the newer professions e.g. primary school teachers, software professionals); (iii) new graduate occupations (where the route has now changed to be via an undergraduate degree e.g. marketing and sales managers, physiotherapists); (iv) niche graduate occupations (where most employees are not graduates but for which there are niches which require graduate skills and knowledge e.g. nurses, retail managers); (v) non-graduate occupations (e.g. receptionist, secretary, carpenter). The first four categories are therefore considered 'graduate level' jobs and the fifth 'non-graduate' level jobs. The fourth category is entitled 'niche graduate occupations' to recognise the fact that the majority of people employed in those jobs do not actually have degrees, although within the job there is likely to be 'ample scope for the exercise of degree level skills and knowledge' (p4).The distinctions are therefore based on a number of factors, but include the 
extent to which graduates are making use of their knowledge and skills. Elias and Purcell validate their categories in a number of ways, including an analysis of the percentage of degree holders in each (which descends as category number ascends). They also present evidence of the changing nature of (at least first destination) graduate occupations from 1995-2002, with a slight but steady increase in the percentage of graduates in what they would classify as 'niche' graduate jobs over the years. Elias and Purcell (2003) showed how graduates tend to migrate from non-graduate to graduate jobs, particularly during the first three years following graduation.

In this paper, we investigate whether a psychology placement year has measurable benefits in terms of career success. Using data from the DLHE survey, we investigate whether placement experience confers any early career benefits. From a survey of psychology alumni, we attempt to discover whether a psychology placement year has any medium term (18 months to two years following graduation) career benefits or whether any early benefits fade over time.

\section{Method}

Anonymous data were obtained from the University electronic records for graduates from 2007 and 2008 which contained DLHE reported employment/ study status 6 months post-graduation and a job ranking from 1 to 5 according to Elias and Purcell (2004) categories and disability status. Salary was reported in some instances.

In addition to the data from the electronic records, we also either emailed (depending on availability of a recent email address) a web link to an online questionnaire or 
sent paper questionnaires to known psychology alumni 2003-2008 asking for measures of career satisfaction, satisfaction with pay and pay level (see Appendix 1 for questionnaire). Up to date contact information was not held for all psychology alumni, but we know that the web link was also passed between graduates via email and social networking sites.

Out of the possible 608 alumni where some (not always accurate) contact information was available, 188 responded to the survey (roughly a $31 \%$ response rate out of those that were and could be asked, or roughly $25 \%$ of the total possible population of alumni for those years). The absolute numbers of responses decreased with increasing time from graduation. This will reflect a number of factors, including (i) increased cohort sizes in the latter years, (ii) loss of contact details over the years, (iii) probable decreased motivation to respond as the connection with the University is weakened. Out of the graduates that responded to the survey, $70 \%$ had taken a placement, which partly reflects the bias toward the percentage of students that had taken placements in that time (58\%). A chi square analysis showed no association between graduation year and whether or not respondents took a placement year, suggesting that any factors influencing response rate were operating roughly equally over the years $(\operatorname{chi}=1.459, \mathrm{df}=5)$. 


\section{Results}

\section{Measuring the early career benefits of a psychology placement year}

Using the DLHE survey data for 201 psychology graduates from 2007 and 2008 graduation years a chi squared analysis showed no association between psychology graduates' placement status and their activity (work/ study/ unemployed/ other) six months post-graduation ( $\mathrm{chi}=3.520, \mathrm{df}=3$, n.s.: see Figure 1 ). In order to investigate the quality or appropriateness of those jobs for the graduates involved, a Kendall's tau analysis was conducted with data from those psychology graduates who were in work to investigate whether there was any association between whether or not they did a placement year and the ranked category (using Elias and Purcell categories) that their job fell into (see Figure 2). Although the data fell in the expected direction i.e. that placement graduates were achieving more highly ranked jobs, the analysis showed no significant relationship between placement status and rank category $(\operatorname{tau}=-0.108)$.

\section{FIGURE 1 ABOUT HERE}

\section{FIGURE 2 ABOUT HERE}

Similar analyses were conducted across the University for 2007 and 2008 graduates of degrees with an optional placement year only. We were able to obtain data for 1507 graduates who fitted this criterion (including the 201 psychology graduates). In this analysis, a chi squared analysis indicated a significant association between graduates' placement status and their activity (work/ study/ unemployed/ other) six months post-graduation ( $c h i=29.28, d f=3, p<0.001)$ : see Figure 3 . Because it was noted that more non-placement students had gone on to further study, a further analysis was conducted investigating only those graduates either in work or 
unemployed - the association remained significant. As before, in order to investigate the quality or appropriateness of those jobs for the graduates involved, a Kendall's tau analysis was conducted with data from those graduates who were in work. This analysis investigated whether there was any association between whether or not they did a placement year and the ranked category (using Elias and Purcell category) that their job fell into (see Figure 4). As with the psychology graduates there was a weak association (tau=-0.070) between placement status and job rank categories. Perhaps at least partly because of the increased numbers of people in this analysis, this relationship was statistically significant $(p<0.05)$. The data fell in the expected direction i.e. that placement graduates were achieving more highly ranked jobs. Whilst $27 \%$ of the placement graduates' jobs would be considered 'nongraduate jobs', $38 \%$ of the non-placement graduates' jobs would be.

\section{FIGURE 3 ABOUT HERE}

\section{FIGURE 4 ABOUT HERE}

However, it is generally accepted that employers prefer graduates with 2.1 or $1^{\text {st }}$ class degrees. As we have shown (e.g. Reddy and Moores, 2006) that doing a placement year increases a student's chance of obtaining a 2.1 degree or above, it would seem prudent to also include this factor in any analysis of employment outcomes. For psychology, analyses of employment outcomes were therefore analysed for 2.1 class and 2.2 class graduates separately. Only graduates with these degree classes and only graduates in work were included in a chi squared analysis to investigate whether there is an association between Elias and Purcell job category (this time collapsed only into graduate vs. non-graduate jobs) and whether or not a 
placement was taken ${ }^{1}$. Rather than attenuating any relationship with the placement year, for the 2.1 classification achieving psychology graduates the association between type of job and placement year was significant (chi=4.41, $\mathrm{df}=1, \mathrm{p}<0.05$ ) with the placement programme graduates obtaining more graduate level jobs. Amongst the students with 2.1 classification degrees $63 \%$ of placement year graduates achieved graduate level jobs within the first six months of graduation, compared to $33 \%$ of the non-placement year graduates. Amongst the graduates who obtained 2.2 degrees this relationship was not apparent and the data actually fell in the opposite direction; $48 \%$ of the placement year graduates had obtained a graduate level job, compared to $65 \%$ of the non-placement year graduates. This pattern was repeated across the university (amongst subjects with an optional placement year), with a significant association between job level and placement status amongst the 2.1 graduates $(\mathrm{chi}=6.73, \mathrm{df}=1, \mathrm{p}<0.01: 76 \%$ vs. $64 \%$ of graduates achieving graduate jobs) but not the 2.2 graduates ( $65 \%$ of placement vs. $62 \%$ of non-placement graduates achieving graduate jobs: $\operatorname{chi}=0.132, \mathrm{df}=1, \mathrm{~ns})$.

It is possible that other pre-existing differences apart from degree classification between students may have contributed to the association between placement status and type of job. Further chi square analyses on the University wide data ${ }^{2}$ investigated whether the relationship between placement status and job status held true when both degree classification and ethnicity were taken into account. Students from some ethnic backgrounds are less likely to take placements so it is important to investigate whether ethnic differences affect both choosing to take a placement (or

\footnotetext{
${ }^{1}$ There were insufficient data for other analyses because of the relatively low number of graduates who receive $1^{\text {st }}$ and $3^{\text {rd }}$ class degrees and the relatively low number reporting themselves as unemployed.

${ }^{2}$ Data were insufficient to conduct the same analysis within psychology only
} 
not) and early career success. Chi square analyses were conducted separately for students awarded 2.1 and 2.2 degree classifications and separately for white vs. students of any other ethnic background (this very broad lumping together of ethnicities necessitated by the small number in some categories) in order to investigate whether there was any association between having taken a placement and the type of job obtained. For both the white and the ethnic minority students who had obtained 2.1 degree classifications, the associations narrowly failed to reach significance ( $c h i=3.37, d f=1, p=0.067$ and $c h i=2.98, d f=1, p=0.084$ respectively). Neither association approached significance for students with 2.2 degree classifications $(\mathrm{chi}=0.024, \mathrm{df}=1$, and $\mathrm{chi}=0.284, \mathrm{df}=1$, respectively).

In terms of reported salary for psychology students 6 months post graduation (in cases where it was reported), there were no significant differences between placement $(£ 16 \mathrm{~K})$ and non-placement $(£ 18 \mathrm{~K})$ graduates $(\mathrm{t}=0.76, \mathrm{df}=107)$. For university students who took degree programmes in which a placement year was optional six months post graduation (in cases where it was reported), there were significant differences between placement (£20K) and non-placement students (£18K): $(t=3.99, d f=763, p<0.001)$. However, on closer inspection of the data, this pattern seemed to be heavily biased by the 114 Business and Management placement year graduates who had reported their salaries (vs. the 3 non-placement year graduates on this programme), which tended to be somewhat higher than the salaries for graduates from other courses. The numbers in each degree class separately were generally too small to make any firm conclusions. 


\section{Measuring the medium term career benefits of a psychology placement year}

The survey of psychology alumni contained questions about both objective and subjective career progress. Questions on career satisfaction $(n=5)$ and career success $(n=4)$ were coded from one (very satisfied/ successful) to five (very dissatisfied/ unsuccessful). Because responses to the different questions on each topic were similar, the scales on career satisfaction (Cronbach's alpha 0.899) and career success (Cronbach's alpha 0.893) were merged. The overall career success measure therefore had a minimum score of 4 (very satisfied on all items) and a maximum score of 20 (very dissatisfied on all items). The overall career satisfaction measure had a minimum score of 5 (very satisfied on all items) and a maximum score of 25 (very dissatisfied on all items).

Career success.

i) Perceived success. A Mann-Whitney $U$ test showed that in terms of overall career success, placement graduates had similar perceived success levels to non-placement graduates $(Z=-1.13$, ns. mean scores 7.6 vs. 8.4 respectively i.e. very satisfied/ satisfied vs. satisfied/ neither satisfied nor unsatisfied). A sequential multiple regression was performed to examine the contribution of different factors to perceived success. At the first step, no significant effects were found for degree classification $(t=1.19$, beta=0.09), ethnicity $(t=1.24$, beta $=0.10)$, gender $(t=0.48$, beta $=0.04)$ or entry year $(t=0.69$, beta $=0.05)$ and together these factors accounted for a non-significant $2.6 \%$ of the variance in 
perceived success. The addition of the placement status variable accounted for only a further, and non-significant, $0.4 \%$ of the variance.

ii) Perceived career schedule. A Kendall's tau analysis was performed on the ordered responses for whether graduates thought their career was (i) ahead of, (ii) on, or (iii) behind schedule and whether or not graduates had taken a placement year. There was a significant association $(\operatorname{tau}=-0.148, p<0.05)$ suggesting that graduates who had taken a placement year were more likely to feel ahead or on schedule in their career compared to their non-placement year counterparts (see Figure 5).

\section{FIGURE 5 ABOUT HERE}

iii) Reported job status. Graduates who were in work were asked whether or not they would have been able to get the job they are doing without the qualification they obtained from Aston University (the qualification, not the subject of study). The possible answers were: a) No - the qualification was a formal requirement/ expected; (b) Possibly - but the qualification did give me an advantage; (c) Yes or (d) Don't know. There was a significant relationship between the answer given and placement status $(\mathrm{Chi}=9.67, \mathrm{df}=3, \mathrm{p}<0.05)$. More placement (68\%) than non-placement graduates (52\%) reported that a degree was a requirement for their current job. In addition to this analysis, graduates were asked a number of questions that also appear on the DLHE survey about their current job (including job title, current duties, company name and number of people in the company). These were coded in exactly the same way as the DLHE survey data into the ranked categories as suggested by Elias and Purcell (see Figure 6). Thus, we produced data 
similar to that created in the DLHE survey six months post graduation except for in follow up format up to six and a half years later. The higher percentages of students in graduate jobs from 18 months to six and a half years following graduation (compared to six months following graduation) reflects the trend described by Elias and Purcell (2003) of graduates migrating to graduate level jobs over time. Although a chi square analysis suggested a significant association between placement status and job category $(\mathrm{chi}=11.00, \mathrm{df}=4$, $p<0.05)$, the Kendall's tau value suggested that this was not a simple or 'linear' relationship (tau=-0.118, ns). Of the placement graduates, $64.2 \%$ had jobs that fell into the first category and $10.8 \%$ in the fifth category. This was in comparison to $54.2 \%$ and $29.2 \%$ of the non-placement graduates. This meant that $30 \%$ of the cells (in no placement job categories two, three and four) had expected values less than five. The job categories were therefore recoded into a simple graduate job (categories one to four) vs. non-graduate job (category five) split. This analysis revealed a significant association between placement status and job type with $89.2 \%$ of placement vs. $70.8 \%$ of non-placement graduates being in graduate jobs (chi=8.54, $\mathrm{df}=1, \mathrm{p}<0.005)$. Although there were insufficient data to conduct separate analyses by graduation year, the difference between placement and non-placement graduates was most evident in the early career stage (graduation year 2008) where 18 months after graduation $75 \%$ of placement graduates but only $30 \%$ of non-placement graduates were in graduate jobs. The association between placement year status and type of job was significant even when examining only those students who had received 2.1 classification degrees (chi=6.85, $d f=1, p<0.01)$ showing that the effect of placement year experience on gaining 
graduate jobs was not related to the academic success of the individuals ( $91.1 \%$ vs. $72.7 \%$ of 2.1 graduates in graduate jobs). Although data were too limited to analyse statistically for 2.2 graduates, similarly to the pattern found six months following graduation, they showed no association between placement status and job type $66.7 \%$ and $69.2 \%$ of placement and nonplacement graduates (respectively) having obtained graduate jobs.

\section{FIGURE 6 ABOUT HERE}

\section{Career satisfaction.}

A Mann-Whitney $U$ test showed that in terms of overall career satisfaction, placement graduates were significantly more satisfied than non-placement graduates $(Z=-2.97, p<0.01$ mean scores 9.17 vs. 11.89 respectively i.e. very satisfied/ satisfied vs. satisfied/ neither satisfied nor unsatisfied). In order to investigate whether the placement year had a significant effect on satisfaction once ethnicity (simplified to white or ethnic minority), gender, degree classification and entry year were taken into account a sequential multiple regression was performed. Degree classification $(t=2.00$, beta $=0.15, p<0.05)$, ethnicity $(t=2.38$, beta $=0.18, p<0.05)$ and entry year $(t=2.41$, beta $=0.18, p<0.05)$ all had significant effects when entered at the first step, but gender $(t=1.00$, beta $=0.07)$ did not. Together these variables accounted for $10.1 \%$ of the variance. Being white, having obtained a better degree classification and having an earlier University entry year were all associated with increased satisfaction. The addition of placement status to the model explained a further $3.8 \%$ of the variance $(p<0.01)$ and its effect was significant $(t=2.77$, beta $=-0.21, p<0.01)$ : having done a placement was associated with increased satisfaction. However, its 
addition to the model rendered entry year and degree classification non-significant $(\mathrm{t}=1.59$, beta $=0.12$ and $\mathrm{t}=1.44$, beta $=0.11$ respectively $)$, ethnicity remained significant $(\mathrm{t}=-2.26$, beta $=0.16, \mathrm{p}<0.05)$ and gender was still not significant $(\mathrm{t}=1.09$, beta $=0.08)$

Salary. Figure 7 shows graduates' reported earnings split by whether or not they took a placement year. Where people had responded with their salary band (120 placement and 46 non-placement graduates) a Mann-Whitney $U$ test was performed on the ordinal level data of the 11 possible salary bands (under $£ 10 K, £ 10-14 K, £ 15$ 20K, £21-25K, £26-30K, £31-35K, £36-40K, £41-45K, £45-50K, £51-55K, more than $£ 55 \mathrm{~K})$. This analysis suggested that placement graduates showed a trend towards earning more than non-placement graduates $(Z=-1.74, p=0.08)$. The placement graduates had a mean band of 4.22 (i.e. in the $21-30 \mathrm{~K}$ range) compared to a nonplacement mean band of 3.65 (i.e. in the 15-25K range). However, a Kendall's tau analysis showed (as might be expected) that graduates earn more with increasing time from graduation $(\mathrm{tau}=-0.399, \mathrm{p}<0.001)$. This was true for both placement $(\mathrm{tau}=-$ 0.376, $\mathrm{p}<0.001)$ and non-placement $(\mathrm{tau}=-0.480, \mathrm{p}<0.001)$ graduates separately.

\section{FIGURE 7 ABOUT HERE}

In order to investigate whether the placement year had a significant effect on salary once ethnicity (simplified to white vs. ethnic minority), gender, degree classification and entry year were taken into account a sequential multiple regression was performed. Entry year $(t=-7.70$, beta $=-0.52, p<0.001)$, had a significant effect when entered at the first step. Degree classification $(t=-1.47$, beta $=-0.10)$, gender $(t=-$ 1.933, beta $=-0.13, p=0.055)$ and ethnicity $(t=0.06$, beta $=0.004)$ did not have 
significant effects. Together these variables accounted for $29.3 \%$ of the variance. The addition of placement status to the model did not have a significant effect, accounting for no extra variance at all. Using graduation year rather than entry year as a variable led to somewhat different results. In this case, graduation year $(t=-7.41$, beta $=-0.50, p<0.001)$, had a significant effect when entered at the first step. The effects of degree classification $(\mathrm{t}=-1.87$, beta $=-0.13, \mathrm{p}=0.06)$ and gender $(\mathrm{t}=-1.88$, beta $=-0.13, p=0.062)$ narrowly missed significance but the effect of ethnicity $(t=-$ 0.13 , beta $=-0.009)$ did not have a significant effect. Together these variables accounted for $27.9 \%$ of the variance. The addition of placement status to the model accounted for a further $1.5 \%$ of the variance $(p=0.07)$. The effect of the placement variable within the model narrowly missed significance $t=1.82$, beta $=0.13, p=0.07$ ). Thus it would seem that whereas taking a placement may have a marginal effect on graduates' salaries when compared to people that graduated in the same year, placement programme graduates do not earn more than the people that started their courses in the same year. Thus, the effect of taking a placement on salary is no greater than a year's experience post-graduation.

Career goals. In terms of graduates knowing and changing their career goals since graduation, placement and non-placement graduates were remarkably similar. Around $57 \%$ of placement and $54 \%$ of non-placement graduates had not changed their career goals since graduation. $22 \%$ vs. $26 \%$ had changed their goals once and $21 \%$ vs. $20 \%$ more than once. A chi square analysis showed the differences to be non-significant (chi=0.323, df=2). 
For $61 \%$ of placement students, the placement was reported to be a step towards their current career. A further $10 \%$ were offered a position by the same employer that they took a placement with. $4.2 \%$ said that their placement position was a step towards the career that they would like to pursue but that they were no further on with that career at the moment. $9.2 \%$ said that the main thing that the placement year taught them was that they did not want to follow that career path.

Thoughts on the placement year. Alumni were asked "Which of these best describes your feelings about how taking (or not taking) a placement year has affected (or not affected) your career progression? ". Of those who did not take a placement year, $18.5 \%$ thought that not taking a placement year had positively affected their career progression in that they were one year further on, $46.3 \%$ thought that it had made no difference to their progression, 33.3\% thought that not taking a placement may have negatively affected their career progression a bit and $1.9 \%$ thought that it had negatively affected their career progression. Of those who did take a placement, $0.8 \%$ thought it had negatively affected their career progression in that they were one year behind where they might have been, $13.7 \%$ reported that it had made no difference and $80.9 \%$ thought it had had a positive effect.

\section{Discussion}

The results show that a psychology placement year has some measurable career benefits at least for some students. Those with 2.1 degree classifications as well as placement experience were more likely to be in graduate level work six months after graduation. However, despite this, the levels of reported salaries at this time point were not modulated by placement experience. Salaries of placement programme graduates in the later survey did show a trend towards being higher than those of 
their non-placement year counterparts, but this difference was no longer significant once a number of background variables (including University entry year) were taken into account, although there was a trend towards placements being associated with higher salaries when graduation year rather than entry year was accounted for. Interestingly, although the groups did not differ in terms of their perceptions of their own success, the placement graduates were less likely to feel that their careers were behind schedule and more likely to have graduate level jobs (even when degree classification was taken into account). Moreover, placement graduates were more satisfied with their careers, even when University entry date (the strictest possible control) and a number of other background factors had been controlled. In summary, therefore, psychology placement experience has some measurable benefits in both the short and medium term, although whether the benefits of the placement year outweigh the costs in monetary terms is inconclusive and depends to some extent on the comparison group used; although placement graduates might be earning marginally more than their peers who they graduated with, they would not be earning more than those that started the degree at the same time as them but chose not to undertake a placement year.

Previous studies e.g. Blasko et al (2002) have found early employment benefits for graduates with substantial periods of work experience, in particular in non-vocational subjects. However, Blasko et al analysed data from a number of different universities and graduates from a number of different subjects. It would therefore appear that many factors in that study were held constant statistically; different Universities have different intakes and offer placement years in different subjects which are not necessarily producing graduates of equal employability to start with 
(regardless of any work placement experience). Thus, although Blasko et al's study had a huge advantage in terms of its sample size and ability to control statistically for a number of factors, we feel that the strength of the career findings from this study is that the graduates were from similar intakes (students can apply for the course either with or without the placement year and are required to achieve the same grades also students can easily swap between courses at any point) and had degrees from the same degree programme in the same university (the only thing that differed was whether or not they chose to take a placement year).

Contrary to at least some of our results, Mason et al (2003) found (in five different University subjects) that any initial benefits of placement participation were no longer significant one to three years later. The long training period to become a chartered psychologist (as discussed in the introduction) may account for the difference between psychology and other subjects at least in terms of pay and progression. Auburn, Ley and Arnold (1993) found that psychology graduates who had taken placements rated their first employment experience as more satisfactory, although commented that this may have been due to cohort differences. Our results suggest greater satisfaction amongst placement programme graduates who graduated in the same year and indeed even amongst those who were matched for entry date. What is not clear is whether (as in the Auburn et al, 2003 paper) this difference in perception is a result of more realistic expectations of work, a genuinely different experience at work or a more appropriate choice of work. In addition, even 18 months to six years after graduation, graduates with placement experience were more likely to be in graduate jobs. 
However, although consistently positive differences are found between placement and non-placement graduates, the differences are not necessarily large. It would appear that placement graduates are certainly not any more than a year ahead in their careers (the year, it could be argued, that they spent doing their placement), but equally, they are not a year behind either (i.e. when their University entry date is considered). Overall, it can be concluded that taking a psychology placement produces some measurable career benefits over both the short and the medium term, but that the placement experience has not been conclusively shown to be either better or worse than the equivalent experience post-graduation, with the possible exception of career satisfaction. Indeed, the main thing that a placement year seems to offer is a springboard into a career and a seemingly 'faster start' into graduate level and better paid jobs as well as achieving greater job satisfaction. Although placement year graduates are getting paid more, they are getting paid similarly when taking their University entry date into account. Proponents of the standard three year degree could therefore argue that a year on slightly lower pay post-graduation is better than a year with very little pay during a placement year, particularly when a proportion of student fees also need to be found. Equally, however, the same support mechanisms (e.g. student loans, parental support) are not always in place post-graduation. It is often the case that placement and nonplacement students have different career goals: as mentioned in the introduction, about half of the placement students take placements in clinical psychology or related fields. It may be that this area is where the real benefit in a psychology placement year lies. In addition, those that do succeed on this career path can ultimately expect to earn a reasonable salary. This is an area for future research. Similarly, more graduates who did not take a placement year go onto further study 
and may be very successful in other areas. Each individual will clearly have to decide for themselves whether a placement year is the right thing to do for them based on career goals, personal choice and financial considerations. Differences between placement and non-placement graduates in perceived job satisfaction are perhaps more important than salary. There are several possible reasons why this might be, including (as above) the type of career choices that graduates have made, or placement students having a slightly more realistic view of working life and the positive and negative experiences it entails. If the latter reason is the case then it might be that the career expectations of graduates need to be managed somewhat. However, overall, our cohort of respondents did seem to be satisfied overall in their careers.

The limitations of this study (and most similar studies) include the reliance on selfreport and the possibility that pre-existing differences between placement and nonplacement students on one or more individual difference dimensions may account for some or all of the benefits. Although in some of the analyses we attempted to control for a limited number of demographic characteristics, it may be differences that are more difficult to measure (such as personality characteristics) can account for the differences. Placement students on the psychology program at Aston University have to apply and be interviewed for placement positions by the potential employers. Although most students are successful in gaining at least one offer of a placement, this is not the case for all students. These students have to go straight into the final year without the placement experience. Similarly, some students will only be offered unpaid experience, when they really wanted paid experience and will choose to return to the final year because of financial constraints, the inherently 'more 
employable' students getting the better placements as well as (later on) the better jobs (only those with fewer financial pressures being able to afford to accept an unpaid placement). There are therefore several factors which can influence whether or not a student takes a placement year, including their own 'employability' preplacement. It is quite possible that differences post-placement reflect little more than differences that were already evident pre-placement. If this is not the case, it is not clear what active ingredients, across a wide range of employment experience, account for the benefits. Perry (1970) offers a developmental model of undergraduate cognitive change and there are also expertise models reflecting a move from novice to expert (Brown, Collins and Duguid, 1989) and enculturation models from a socio-cultural perspective (Lave, 1988). King and Kitchener (2002) suggest that, deriving from Perry's work, three assumptions can be made; that students' approach to learning is affected by their understanding of the nature of knowledge; that this understanding changes in a developmentally ordered fashion over time; and that it is also tied to a developing sense of self. They suggest that development may be aided in a number of ways, notably by having ill-structured issues to discuss and analyse and by having varied settings to practice and gain confidence in thinking skills. It is possible that placements offer this scaffolding to cognitive development. Investigating this further may pin down key factors in work placements and also reveal elements that are missing in undergraduate education outside of placements. 


\section{References}

Auburn, T., Ley, A. and Arnold, J.(1993). Psychology undergraduates' experience of placements: a role-transition perspective. Studies in Higher Education, 18, 265285.

Blasko, Z. with Brennan, J., Little, B. and Shah, T. (2002). Access to what: an analysis of factors determining graduate employability. Bristol: Higher Education Funding Council for England.

Brown, J., Collins, A. and Duguid, P. (1989). Situated cognition and the culture of learning. Educational Researcher, January-February, 1989, 32-42.

Cranmer, S. (2006). Enhancing graduate employability: best intentions and mixed outcomes. Studies in Higher Education, 31, 169-184.

Elias, P. and Purcell, K (2004) SOC(HE): a classification of occupations for studying the graduate labour market Graduate Careers Seven Years On Research Paper No.6 IER Warwick/ESRU UWE.

Elias, P. and K. Purcell (2003) Measuring change in the graduate labour market. A study of the changing nature of the labour market for graduates and high level skills. Graduate Careers Seven Years On Research Paper No.1, IER Warwick/ESRU UWE.

Greenhaus, J.H., Parasuraman, S. and Wormley, W.M. (1990). Effects of race on organizational experiences, job performance evaluations, and career outcomes. The Academy of Management Journal, 33, 64-86.

Harvey, L., Moon, S., and Geall, V. with Bower, R. (1997). Graduates' work: organisational change and students' attributes. Birmingham: Centre for 
Research into Quality, University of Central England, Birmingham and The Association of Graduate Recruiters.

King, P. and Kitchener, K. (2002). The reflective judgement model: twenty years of research on epistemic cognition, in B. K. Hofer and P. R. Pintrich (eds.) Personal epistemology: the psychology of beliefs about knowledge and knowing; (pp. 27-61), New York, Routledge.

Lave, J. (1988). Cognition in practice; mind, mathematics and culture in everyday life. Cambridge, Cambridge University Press.

Mason, G., Williams, G., Cranmer, S. And Guile, D.(2003). How much does higher education enhance the employability of graduates? Bristol: Higher Education Funding Council for England.

Perry, W. G. Jr. (1970). Forms of intellectual and ethical development in the college years: A scheme. New York, Holt, Rinehart and Winston

Reddy, P. and Moores, E. (2006). Measuring the benefits of a psychology placement year. Assessment \& Evaluation in Higher Education, 31, 551-567.

Turban, D.B. and Dougherty, T.W. (1994). Role of protégé personality in receipt of mentoring and career success. The Academy of Management Journal, 37, 688702. 
Figure 1. DLHE survey data of graduates (2007-2008) who had the opportunity to take an optional placement year: reported activities six months post-graduation

\begin{tabular}{|l|c|l|l|c|}
\hline & \multicolumn{4}{|c|}{ Activity } \\
\hline & Work & Unemployed & Study only & Other \\
\hline Placement & $868(77.1 \%)$ & $63(5.6 \%)$ & $124(11.0 \%)$ & $71(6.3 \%)$ \\
\hline No placement & $250(65.6 \%)$ & $47(12.3 \%)$ & $62(16.3 \%)$ & $22(5.8 \%)$ \\
\hline
\end{tabular}

Figure 2. Graduates (2007-8) in work: a breakdown of the job categories by placement year.

\begin{tabular}{|l|c|c|c|c|c|}
\hline \multirow{2}{*}{} & \multicolumn{5}{|c|}{ Elias and Purcell Job Category } \\
\cline { 2 - 6 } & $\mathbf{1}$ & $\mathbf{2}$ & $\mathbf{3}$ & $\mathbf{4}$ & $\mathbf{5}$ \\
\hline Placement & $76(8.8 \%)$ & $118(13.6 \%)$ & $244(28.1 \%)$ & $197(22.7 \%)$ & $233(26.8 \%)$ \\
\hline No placement & $23(9.2 \%)$ & $31(12.4 \%)$ & $48(19.2 \%)$ & $54(21.6 \%)$ & $94(37.6 \%)$ \\
\hline
\end{tabular}

Figure 3. DLHE survey data of psychology graduates (2007-2008): reported activities six months post-graduation

\begin{tabular}{|l|c|l|l|l|}
\hline & \multicolumn{4}{|c|}{ Activity } \\
\hline & \multicolumn{1}{|c|}{ Work } & Unemployed & Study only & Other \\
\hline Placement & $99(75 \%)$ & $7(5.3 \%)$ & $22(16.7 \%)$ & $4(3 \%)$ \\
\hline No placement & $47(68.1 \%)$ & $5(7.2 \%)$ & $11(15.9 \%)$ & $6(8.7 \%)$ \\
\hline
\end{tabular}

Figure 4. Psychology graduates (2007-2008) in work: a breakdown of the job categories by placement year

\begin{tabular}{|l|c|c|c|c|c|}
\hline \multirow{2}{*}{} & \multicolumn{5}{|c|}{ Elias and Purcell Job Category } \\
\cline { 2 - 6 } & $\mathbf{1}$ & $\mathbf{2}$ & $\mathbf{3}$ & $\mathbf{4}$ & $\mathbf{5}$ \\
\hline Placement & $20(20.2 \%)$ & $11(11.1 \%)$ & $14(14.2 \%)$ & $15(15.2 \%)$ & $39(39.4 \%)$ \\
\hline No placement & $4(8.5 \%)$ & $4(8.5 \%)$ & $7(14.9 \%)$ & $11(23.4 \%)$ & $21(44.7 \%)$ \\
\hline
\end{tabular}


Figure 5. Graph to show percentage of placement vs. non-placement year graduates who believe that their career in on, behind or ahead of schedule

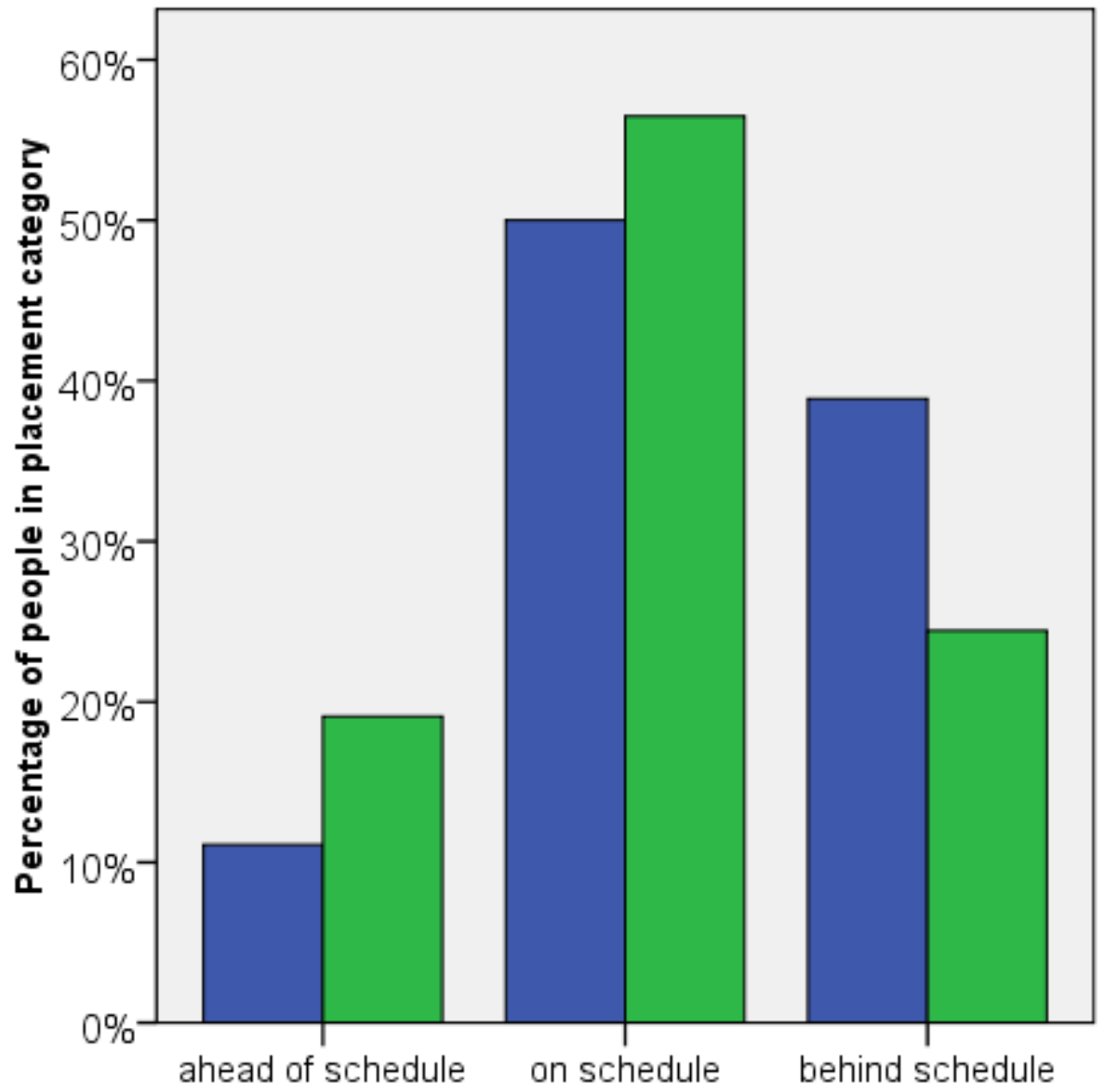

$\square$ no placement

$\square$ placement

Figure 6. Graduates (2003-2008) in work: a breakdown of the job categories by placement year.

\begin{tabular}{|l|c|c|c|c|c|}
\hline \multirow{2}{*}{} & \multicolumn{5}{|c|}{ Elias and Purcell Job Category } \\
\cline { 2 - 6 } & $\mathbf{1}$ & $\mathbf{2}$ & $\mathbf{3}$ & $\mathbf{4}$ & $\mathbf{5}$ \\
\hline Placement & $77(73.6 \%)$ & $6(5.0 \%)$ & $11(10.7 \%)$ & $13(10.8 \%)$ & $13(10.8 \%)$ \\
\hline No placement & $26(54.2 \%)$ & $3(6.2 \%)$ & $4(8.3 \%)$ & $1(2.1 \%)$ & $14(29.2 \%)$ \\
\hline
\end{tabular}


Figure 7. Graph to show percentage of placement vs. non-placement year graduates earnings

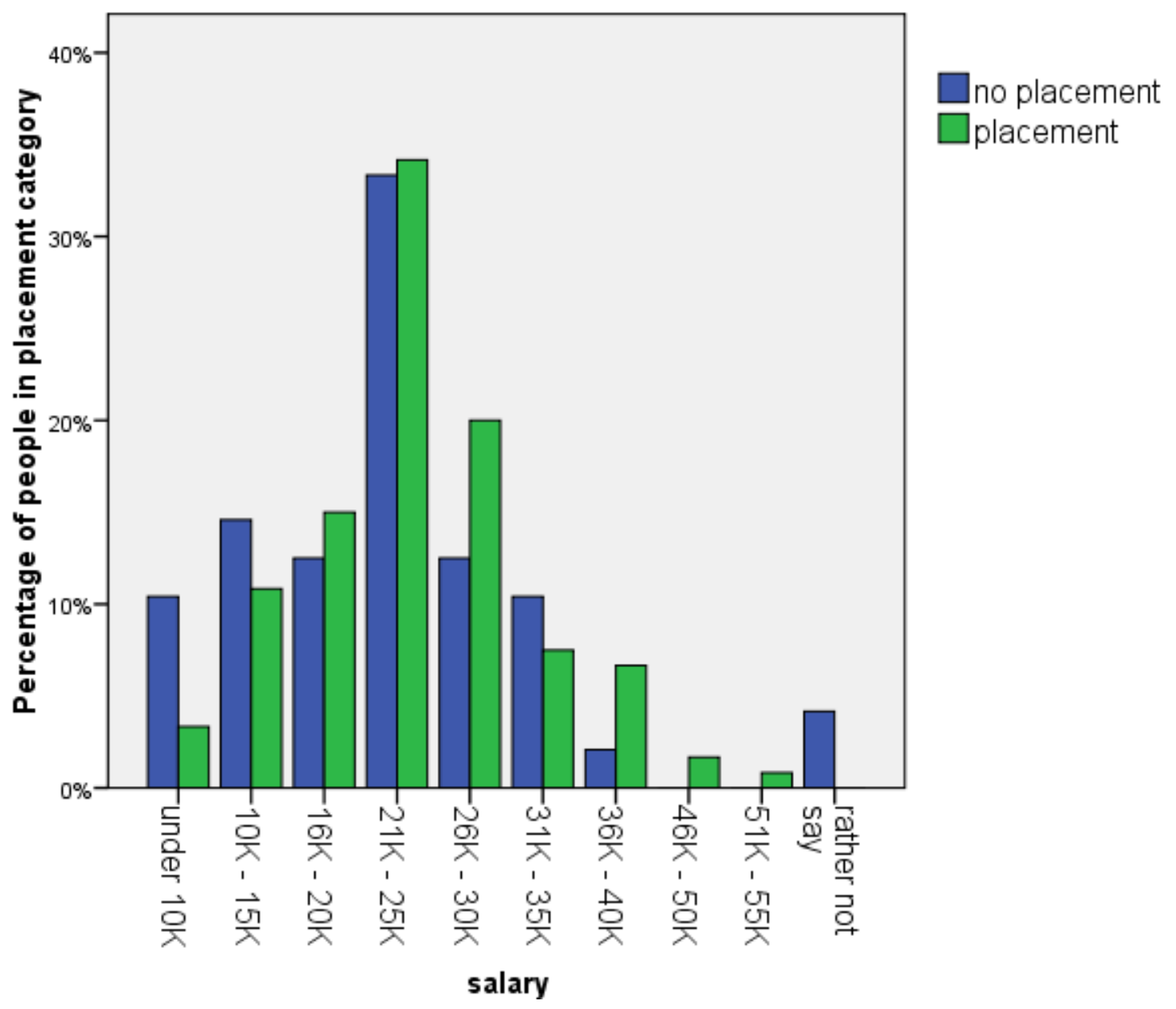


Appendix 1. Questionnaire

Basic information:

1. What year did you graduate?

2. What class of degree were you awarded:
a) $1^{\text {st }}$
d) $3^{\text {rd }}$
b) 2.1
e) Pass
c) 2.2
f) Unclassified

3. Was it possible to take a placement year in your degree programme?
a) Yes
b) No
c) Don't know

4. Did you take a placement year? Yes/No

5. Do you have a disability? Yes/ No/ Rather not say

6. Which of these categories best describes your ethnicity:
a) White (e.g. white British, white Irish or any other white background)
b) Mixed (e.g. white and black Caribbean, white and black African, white and Asian or any other mixed background)
c) Black or Black British (e.g. Caribbean, African, any other Black background)
d) Asian or Asian British
e) Chinese or other ethnic group
f) Rather not say

7. Date of birth

8. Gender : Male/ Female

\section{Perceptions of your career so far}

9. How successful has your career been so far?
a) Very successful
b) Moderately successful
c) Neither successful nor unsuccessful
d) Moderately unsuccessful
e) Very unsuccessful

10. Compared to your co-workers, how successful is your career?
a) Very successful
b) Moderately successful
c) Neither successful nor unsuccessful
d) Moderately unsuccessful
e) Very unsuccessful 
11.Compared to your University peers, how successful is your career?
a) Very successful
b) Moderately successful
c) Neither successful nor unsuccessful
d) Moderately unsuccessful
e) Very unsuccessful

12. How successful do your 'significant others' (e.g. partners, parents, friends) feel your career has been:
a) Very successful
b) Moderately successful
c) Neither successful nor unsuccessful
d) Moderately unsuccessful
e) Very unsuccessful

13.I am satisfied with the success I have achieved in my career
a) strongly agree
b) agree to some extent
c) uncertain
d) disagree to some extent
e) strongly disagree

14. I am satisfied with the progress I have made toward meeting my overall career goals
a) strongly agree
b) agree to some extent
c) uncertain
d) disagree to some extent
e) strongly disagree

15.I am satisfied with the progress I have made toward meeting my goals for income
a) strongly agree
b) agree to some extent
c) uncertain
d) disagree to some extent
e) strongly disagree

16.I am satisfied with the progress I have made toward meeting my goals for advancement
a) strongly agree
b) agree to some extent
c) uncertain
d) disagree to some extent
e) strongly disagree 
17.I am satisfied with the progress I have made toward meeting my goals or the development of new skills
a) strongly agree
b) agree to some extent
c) uncertain
d) disagree to some extent
e) strongly disagree

18. Given your age, do you think that your career is:
a) on schedule
b) ahead of schedule
c) behind schedule

19. Have you now got the job or career that you ultimately want?
a) Yes, it's great!
b) Not yet, but I am in training for it now
c) No, but the work I am doing is a step towards it
d) No, but I am happy with what I am doing for the moment
e) No, this is not what I want to do in the long run

20. If you haven't got the job or career that you ultimately want, do you know the career direction that you want to go in?
a) Yes, I know the career that I want
b) When I graduated I had a clear direction but now I am not sure
c) Possibly, I am not sure yet
d) Not yet, I am still exploring
e) I already have the career or job that I want

21. Which of these best describes your feelings about how taking (or not taking) a placement year has affected (or not affected) your career progression?
a) Not taking a placement year
has positively affected my
career progression: I am one
year further on
b) Not taking a placement year
has not really made any
difference to my career
progression
c) Not taking a placement year
may have negatively affected
my career progression a bit
d) Not taking a placement year
has negatively affected my
career progression
e) Taking a placement year has negatively affected my career progression: I am one year behind where I could have been
f) Taking a placement year has not really made any difference to my career progression
g) Taking a placement year may have had a negatively affected my career progression a bit
h) Taking a placement year has positively affected my career progression




\section{A bit about your career so far:}

22. What is your current activity/ employment status:
a) Full time paid work only
b) Part time paid work only
c) Self-employed/ freelance
d) Voluntary/ unpaid work only
e) Permanently unable to work/ retired
f) Temporarily sick or unable to work/ looking after the home or family
g) Taking time out in order to travel
h) Due to start a job within the next month
i) Unemployed and looking for employment, further study or training
j) Unemployed and NOT looking for employment, further study or training
k) Doing paid work and further study
I) Doing further study only - full time
m) Doing further study only - part time
n) Doing something else
o) Rather not say

23. Please give brief details of training, paid or voluntary work since graduation and whether you consider each job 'graduate level' (Yes/No)

\begin{tabular}{|l|l|l|l|}
\hline $\begin{array}{l}\text { From } \\
\text { (month/ } \\
\text { year) }\end{array}$ & $\begin{array}{l}\text { To } \\
\text { (month/ } \\
\text { year) }\end{array}$ & Job title & $\begin{array}{l}\text { Graduate } \\
\text { level job } \\
\text { (Y/N) }\end{array}$ \\
\hline & & & \\
\hline & & & \\
\hline & & & \\
\hline & & & \\
\hline & & & \\
\hline & & & \\
\hline
\end{tabular}

24. Have your career goals changed since you graduated?
a) Yes, more than once (please describe below)
b) Yes, once (please describe below)
c) No 
If you are currently in work:

25. What is your current job title?

26. Briefly describe your duties e.g. maintaining and updating company internet

27. What is the name of the organisation you are working for?

28. Approximately how many people work in the entire organisation (i.e. all branches, depts. etc.)?
a) 1 to 49
b) 50 to 249
c) 250 or more
d) I don't know

29. Would you have been able to get the job you are doing without the qualification you obtained from Aston University (the qualification, not the subject of study)?
a) No - the qualification was a formal requirement/ expected
b) Possibly - but the qualification did give me an advantage
c) Yes
d) Don't know

30. Please indicate your annual salary before tax. If you were employed for less than a year or were part time, please estimate your pay to the full time equivalent:
a) Under $£ 10,000$
b) $£ 10$ to $15 \mathrm{k}$
c) $£ 16$ to $20 \mathrm{k}$
d) $£ 21$ to $25 \mathrm{k}$
e) $£ 26$ to $30 k$
f) $£ 31$ to $35 \mathrm{k}$
g) $£ 36$ to $40 \mathrm{k}$
h) $£ 41$ to $£ 45 \mathrm{k}$
i) $£ 46$ to $£ 50 \mathrm{k}$
j) $£ 51$ to $£ 55 \mathrm{k}$
k) More than $£ 55 \mathrm{k}$
l) Rather not say 
31. Which of the following best describes the relationship between your placement and your current employment:
a) I did not take a placement year
b) My placement position was unrelated to my current career
c) My placement position was a step towards my current career
d) The main thing my placement year taught me was that I didn't want to follow that career path
e) My placement position was a step towards the career that I would like to pursue but I am no further on with that career at the moment
f) I was offered a position by the same employer that I took a placement with

